\title{
High-power fiber laser with real-time mode switchability
}

\author{
Hanshuo Wu (吴函炼) ${ }^{1}$, Jiangtao Xu (徐江韬) ${ }^{2}$, Liangjin Huang (黄良金) ${ }^{1}$, Xianglong Zeng (曾祥龙) ${ }^{2}$, and \\ Pu Zhou (周 朴) $)^{*}$ \\ ${ }^{1}$ College of Advanced Interdisciplinary Studies, National University of Defense Technology, Changsha 410073, China \\ ${ }^{2}$ Key Laboratory of Specialty Fiber Optics and Optical Access Networks, Shanghai University, Shanghai 200444, China \\ *Corresponding author: zhoupu203@163.com \\ Received August 29, 2021 | Accepted October 21, 2021 | Posted Online November 15, 2021
}

\begin{abstract}
A hundred-watt-level spatial mode switchable all-fiber laser is demonstrated based on a master oscillator power amplifier scheme. The performance of the amplifier with two seed lasers, i.e., with the acoustically induced fiber grating (AIFG) mode converter inside and outside the seed laser cavity, is investigated. Real-time mode switching with millisecond scale switching time between the $L P_{01}$ and $L P_{11}$ modes while operating in full power $(>100 \mathrm{~W}$ ) is realized through an AIFG driven by radio frequency modulation. This work could provide a good reference for realizing high-power agile mode switchable fiber lasers for practical applications.
\end{abstract}

Keywords: acoustically induced fiber grating; high power; fiber amplifier; mode switching.

DOI: 10.3788/COL202220.021402

\section{Introduction}

Fiber lasers have already found a wide variety of applications ranging from scientific research and medical treatment to industrial processing ${ }^{[1-8]}$, owing to their outstanding characteristics of high-power capability, system simplicity, easy thermal management, etc. ${ }^{[6]}$. In recent years, fiber lasers with dynamic spatial mode profiles have attracted extra attention for their specific advantages in material processing. For instance, several companies have individually launched commercially available mode adjustable/switchable fiber lasers for industrial processing $^{[9-11]}$, the beam profiles of which could be adjusted according to the processing stages and application scenarios for improved processing quality.

In fact, there are several approaches to realizing mode adjusting/switching in fiber lasers ${ }^{[12]}$, and technically an 'optical switch' combined with mode selective components should be adopted. The mode switching/adjusting operation could either be realized by real optical components, such as acoustic-optic tunable filter $(\mathrm{AOTF})^{[13]}$, special fiber coupler ${ }^{[14-17]}$, acoustically induced fiber grating (AIFG) ${ }^{[18-21]}$, polarization controller $(\mathrm{PC})^{[22-27]}$, and spatial light modulator ${ }^{[28]}$, or by some physical mechanisms, such as temperature control of few-mode fiber Bragg gratings $(\mathrm{FBGs})^{[29]}$, altering the excitation condition $^{[30]}$, and injection locking ${ }^{[31]}$. However, fast mode switching between different transverse modes in fiber lasers has only been demonstrated using AOTF and AIFG. In 2013, Daniel et al. demonstrated a 5-W-level mode switchable Tm-doped fiber laser operating around $1921 \mathrm{~nm}$, where the mode switching between the $\mathrm{LP}_{01}$ and $\mathrm{LP}_{11}$ modes is realized by an AOTF and a few-mode fiber grating ${ }^{[13]}$. The whole system was based on a bulk configuration, and the mode switching speed reached an approximately kilohertz $(\mathrm{kHz})$ level. In $2018, \mathrm{Lu}$ et al. experimentally demonstrated fast switchable generation of $\mathrm{LP}_{11 a / b}$ modes and optical vortex beams with \pm 1 st-order orbital angular momentum (OAM) in an all-fiber Er-doped fiber laser, and switching speed up to $4.3 \mathrm{kHz}$ was realized through an AIFG converter ${ }^{[18]}$. In 2019, we reported an $\mathrm{LP}_{01}$ and $\mathrm{LP}_{11}$ mode switchable fiber laser operating at $1070 \mathrm{~nm}$ with $\sim 6 \mathrm{~W}$ output power aided by an $\mathrm{AIFG}^{[32]}$. Complete mode switching at the rate of $250 \mathrm{~Hz}$ and partial mode switching at the rate of $1 \mathrm{kHz}$ were demonstrated. Note that, in the above-mentioned mode switchable fiber lasers based on AIFG, the single-mode rare-earth-doped fiber is used as the gain medium, and, therefore, the gain is extracted by the fundamental mode even when generating a high-order mode laser. Since the situation is more complicated in a few-mode rare-earth-doped fiber owing to transverse mode competition, it is worth studying whether the AIFG could function well in a real few-mode fiber oscillator. Moreover, almost all of the reported mode switchable fiber lasers based on AIFG operate in the low power regime, and their potential for further power scaling is still to be explored.

In this Letter, we demonstrate a hundred-watt-level spatial mode switchable fiber laser system adopting an AIFG. The output beam profiles can be switched from the $\mathrm{LP}_{01}$ mode to the $\mathrm{LP}_{11}$ mode as well as a mixture of both modes during full power operation by varying the applied modulation frequency on the AIFG. The performance, including mode switching speed, beam profile maintenance, output power, and overall efficiency, of the mode switchable fiber laser system exploiting two different seed lasers is systematically investigated. 


\section{Experimental Setup}

The experiment system is based on a master oscillator power amplifier (MOPA) structure, which consists of a mode switchable seed fiber laser operating at $\sim 1070 \mathrm{~nm}$ and a single-stage fiber amplifier. The fiber amplifier includes a $(2+1) \times 1$ pump and signal combiner (PSC), two $976 \mathrm{~nm}$ multimode laser diodes (LDs), a piece of 5-m-long ytterbium-doped fiber (YDF), and a quartz block holder $(\mathrm{QBH})$. The output power of the two LDs is combined by the PSC, and the total pump power reaches $\sim 120.3 \mathrm{~W}$. The core/cladding diameter of the signal arms of the PSC, the pigtail fiber of the QBH, and the YDF is all 15/ $130 \mu \mathrm{m}$. The absorption coefficient of the YDF is $\sim 5 \mathrm{~dB} / \mathrm{m}$ at $976 \mathrm{~nm}$. Two different kinds of seed laser sources are adopted in this setup. The first one is based on a conventional singlemode fiber oscillator, the output port of which is connected with an AIFG before splicing with the fiber amplifier, as shown in Fig. 1(a). The second one has the AIFG integrated within the cavity. As shown in Fig. 1(b), the fiber oscillator is composed of a single-mode fiber loop mirror (FLM), a mode field adapter (MFA), an AIFG, a $(2+1) \times 1$ PSC, a piece of 5-m-long YDF, and a few-mode FBG. The parameters of the YDF are the same as the YDF used in the fiber amplifier. The FLM is implemented by splicing the adjacent two ports of a $3 \mathrm{~dB}$ coupler, and the core/ cladding diameter of the pigtail fiber is $10 / 125 \mu \mathrm{m}$. The core/ cladding diameter of the germanium-doped fiber (GDF) in the AIFG is $15 / 130 \mu \mathrm{m}$, and the MFA is spliced between the AIFG and the FLM to decrease the insertion loss. A PC is integrated between the AIFG and the PSC to help control the propagating mode within the cavity. Cladding light strippers (CLSs), which are made by coating high refractive index glue on the bare fiber around the splicing point between the YDF and QBH as well as the splicing point between the YDF and the FBG, are used to remove the cladding mode. Note that the $V$ number of all of

(a)

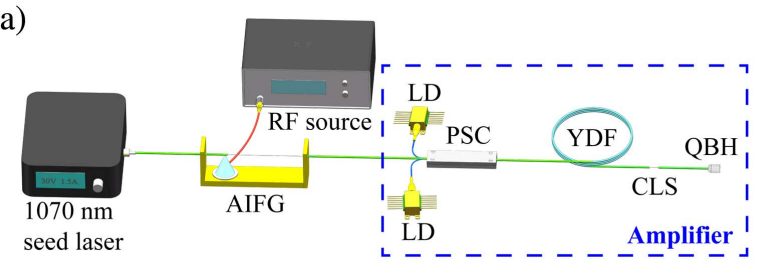

(b)

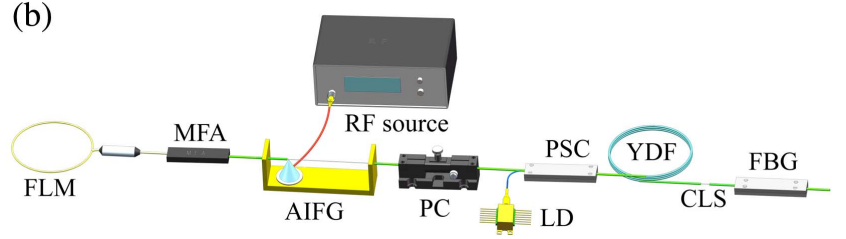

Fig. 1. (a) Experimental setup of the mode switchable fiber laser based on a master oscillator power amplifier scheme; (b) schematic of the intra-cavity mode switchable seed fiber laser. AIFG, acoustically induced fiber grating; RF, radio frequency; LD, laser diode; PSC, pump and signal combiner; YDF, ytterbium-doped fiber; CLS, cladding light stripper; $\mathrm{QBH}$, quartz block holder; FLM, fiber loop mirror; MFA, mode field adapter; PC, polarization controller; FBG, fiber Bragg grating. the $15 / 130 \mu \mathrm{m}$ fiber is $\sim 3.52$ at $1070 \mathrm{~nm}$; therefore, only the two lowest-order modes, i.e., $\mathrm{LP}_{01}$ and $\mathrm{LP}_{11}$ modes, are supported. Moreover, the amplifier of the second scheme is identical to the first scheme shown in Fig. 1(a).

The few-mode FBG and the AIFG are first characterized with the methods illustrated in our previous work ${ }^{[32]}$, and the characterization results are shown in Fig. 2. The few-mode FBG is written on a piece of photosensitive $15 / 130 \mu \mathrm{m}$ GDF, and the measured transmission/reflection spectra of the FBG are depicted in Fig. 2(a), where the two reflection peaks corresponding to the $\mathrm{LP}_{11}$ and $\mathrm{LP}_{01}$ modes of the FBG locate at $1068.71 \mathrm{~nm}$ and $1069.28 \mathrm{~nm}$, respectively. The reflectivity of this FBG is about $\sim 43 \%$ at $1070 \mathrm{~nm}$, with a $3 \mathrm{~dB}$ bandwidth of $0.25 \mathrm{~nm}$. It should be noted that the relative intensity of the reflection peaks does not represent the reflectivity of the $\mathrm{LP}_{01}$ and $\mathrm{LP}_{11}$ modes, and technically these two modes should have very similar reflectivity ${ }^{[33]}$. As for the AIFG, the acoustic-light interaction length is $0.45 \mathrm{~m}$ long, which is defined by the length of the unjacketed GDF. Figure 2(b) shows the measured transmission spectra of the AIFG under the loaded modulation frequencies of $697.70 \mathrm{kHz}$ and $698.52 \mathrm{kHz}$. The applied peak-peak voltage on the AIFG is optimized to be $290.5 \mathrm{~V}$. The two transmission dips locate at 1068.70 and $1070.06 \mathrm{~nm}$, indicating where the mode conversion happens. The depths of the transmission
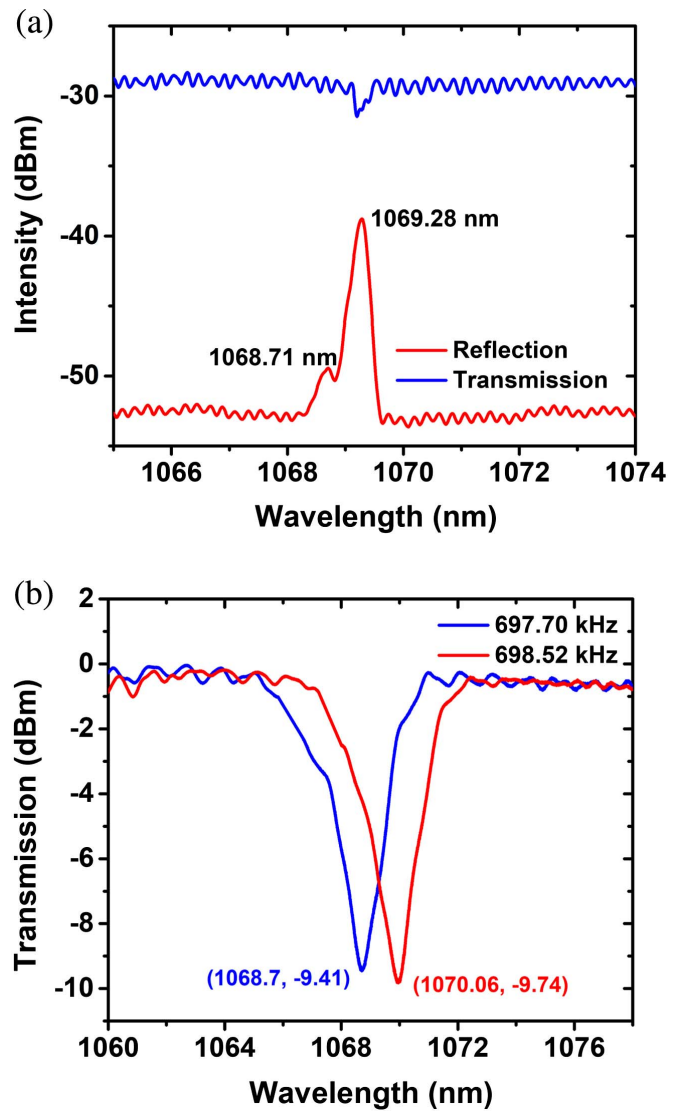

Fig. 2. (a) Transmission and reflection spectra of the few-mode FBG used in the intra-cavity mode switchable fiber oscillator; (b) transmission spectra of the AIFG under different modulation frequencies. 
dips are $9.41 \mathrm{~dB}$ and $9.74 \mathrm{~dB}$, respectively, which correspond to the mode conversion efficiency of $\sim 88.54 \%$ and $\sim 89.38 \%$ from the $\mathrm{LP}_{01}$ to $\mathrm{LP}_{11}$ mode. The modulation frequency $(698.52 \mathrm{kHz}$ for $1070 \mathrm{~nm}$ ) deviates from the theoretical value $(674.044 \mathrm{kHz})$, which possibly results from the residual strain in the unjacketed GDF during the AIFG fabrication ${ }^{[34]}$.

\section{Results and Discussion}

As for the MOPA shown in Fig. 1(a), the output mode from the $1070 \mathrm{~nm}$ seed laser could be manipulated by actively controlling the applied RF signal on the AIFG. The output mode would be switched from the $\mathrm{LP}_{01}$ mode to the $\mathrm{LP}_{11}$ mode upon applying the RF signal of $698.52 \mathrm{kHz}$. The output power of the seed laser is $\sim 8.5 \mathrm{~W}$ in the $\mathrm{LP}_{01}$ mode and decreases to $\sim 6.8 \mathrm{~W}$ as the operating mode switches to the $\mathrm{LP}_{11}$ mode. The power degradation results from the dissipated cladding mode power after mode conversion (referring to 'conversion loss'). Power scaling of the seed laser is subsequently conducted in the fiber amplifier. The output power of the $\mathrm{LP}_{01}$ and $\mathrm{LP}_{11}$ modes from the amplifier as a function of the pump power is shown in Fig. 3(a). The maximum output power reaches $105.7 \mathrm{~W}$ for the $\mathrm{LP}_{01}$ mode
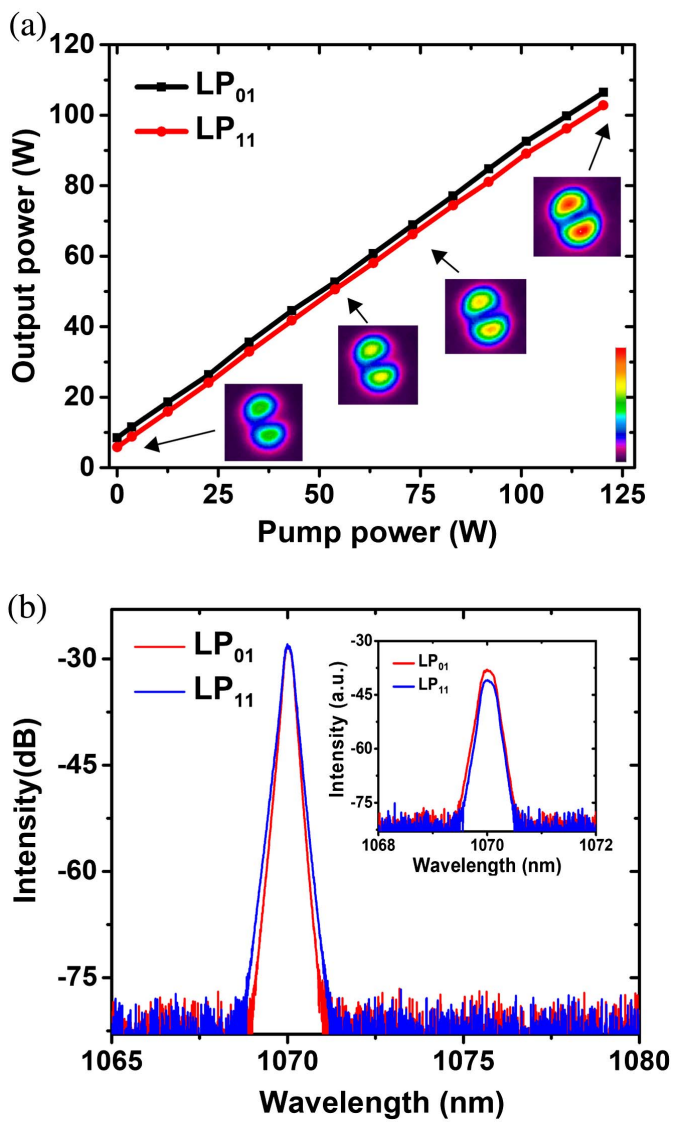

Fig. 3. (a) Output power of the $L P_{01}$ and $L P_{11}$ modes from the fiber amplifier as a function of the pump power (inset: beam profiles of the $L P_{11}$ mode); (b) output spectra of the $L P_{01}$ and $L P_{11}$ modes under the maximum output power (inset: output spectra of the seed laser). with a slope efficiency of $\sim 80.80 \%$ and $101.3 \mathrm{~W}$ for the $\mathrm{LP}_{11}$ mode with a slope efficiency of $\sim 78.55 \%$ under the maximum pump power of $120.3 \mathrm{~W}$. Further power scaling is limited by the available pump power. The output beam profiles of the $\mathrm{LP}_{11}$ mode under different pump powers are recorded and depicted in Fig. 3(a), which are well maintained during the power scaling process, indicating the high purity of the output mode. The output spectra of both modes under the maximum output power are shown in Fig. 3(b), which broaden slightly as the pump power increases. The $3 \mathrm{~dB}$ linewidth of the $\mathrm{LP}_{01}$ mode laser increases from $\sim 0.23$ to $\sim 0.25 \mathrm{~nm}$, while that of the $\mathrm{LP}_{11}$ mode increases from $\sim 0.23$ to $\sim 0.27 \mathrm{~nm}$. The slightly different linewidth broadening phenomenon is possibly owing to the dispersion difference of these two modes.

Furthermore, as shown in Fig. 1(b), an intra-cavity mode switchable seed fiber oscillator is built by integrating an AIFG within the cavity. In previous work based on $\operatorname{AIFG}^{[18,32]}$, where a piece of single-mode YDF is used, the gain is extracted by the fundamental mode regardless of the output mode. Here, a piece of few-mode YDF is used to provide the gain, it is designed that the YDF is directly connected with the output coupling FBG, and, therefore, the gain in this laser is extracted by the output mode. Moreover, the pigtail fiber of the $3 \mathrm{~dB}$ coupler could only support the fundamental mode, thus ensuring pure fundamental mode injection into the AIFG, and a PC is placed between the AIFG and the PSC to further improve the mode purity. Upon applying the RF signal of $697.70 \mathrm{kHz}$ on the AIFG, the output wavelength shifts from 1069.28 to $1068.71 \mathrm{~nm}$, while the output mode switches from the $\mathrm{LP}_{01}$ to $\mathrm{LP}_{11}$ mode at the same time. The output power of both modes as a function of the pump power is presented in Fig. 4(a). The output power reaches $\sim 7.22 \mathrm{~W}$ in the $\mathrm{LP}_{01}$ mode and $\sim 5.94 \mathrm{~W}$ in the $\mathrm{LP}_{11}$ mode under the pump power of $\sim 14.52 \mathrm{~W}$. The slope efficiencies of the $\mathrm{LP}_{01}$ and $\mathrm{LP}_{11}$ modes are $\sim 49.72 \%$ and $\sim 40.91 \%$, respectively. The relatively low slope efficiency could be attributed to the relatively high reflectivity of the output coupling FBG ( $43 \%)$ and the insertion loss of the $3 \mathrm{~dB}$ coupler. Moreover, when the laser operates in the $\mathrm{LP}_{11}$ mode, upon the reflected $\mathrm{LP}_{11}$ mode passing through the AIFG and going into the single-mode fiber, part of the unconverted $\mathrm{LP}_{11}$ mode would be dissipated in the single-mode fiber, and, in combination with the conversion loss, further decreases the lasing efficiency. Except for the sole $\mathrm{LP}_{01 / 11}$ mode operation, hybrid mode operation of the $\mathrm{LP}_{01}$ and $\mathrm{LP}_{11}$ modes manifesting a dual-wavelength output spectrum could also be obtained by tuning the applied modulation frequency between $696.32 \mathrm{kHz}$ and $699.71 \mathrm{kHz}$. An illustration of output beam profiles as well as the corresponding output spectra is shown in Fig. 4(b). The beam profiles of the $\mathrm{LP}_{01}$ and $\mathrm{LP}_{11}$ modes could be well preserved in this fiber oscillator while increasing the pump power, however, the output beam profile would vary when the laser operates in the hybrid mode owing to the dynamic mode competition during the power scaling process.

Power scaling of the intra-cavity mode conversion seed fiber laser by MOPA is also performed. The seed power of $7.22 \mathrm{~W}$ in the $\mathrm{LP}_{01}$ mode and $5.94 \mathrm{~W}$ in the $\mathrm{LP}_{11}$ mode are individually 
(a)

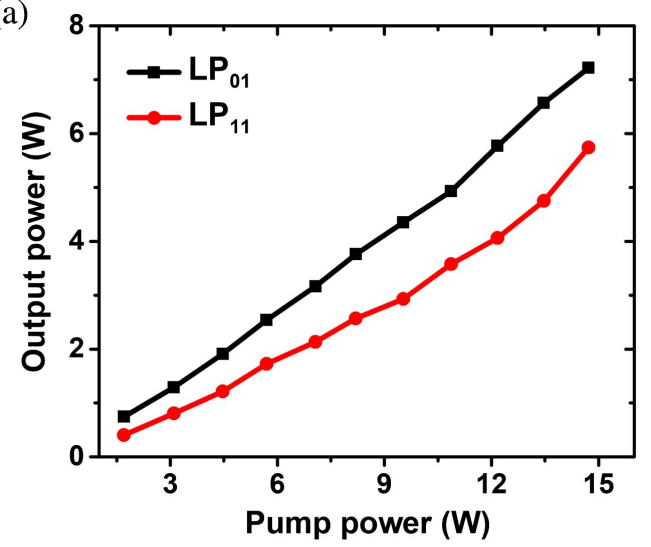

(b)

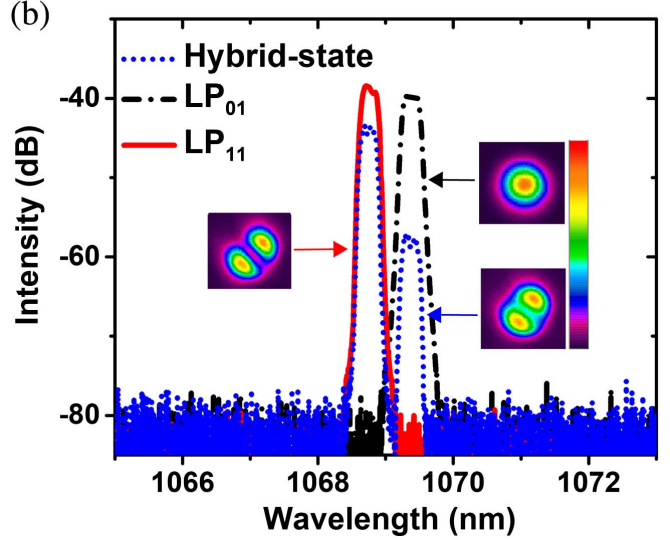

Fig. 4. (a) Output power of the $L P_{01}$ and $L P_{11}$ modes from the fiber oscillator as a function of the pump power; (b) output spectra and corresponding beam profiles of different operation modes.

injected into the fiber amplifier, and the results are shown in Fig. 5. The output powers of the $\mathrm{LP}_{01}$ and $\mathrm{LP}_{11}$ modes both increase linearly with the pump power with very similar slope efficiencies of $\sim 80.95 \%$ and $\sim 78.69 \%$, respectively. The corresponding maximum output power of the $\mathrm{LP}_{01}$ and $\mathrm{LP}_{11}$ modes reaches 104.6 and $100.6 \mathrm{~W}$ under the maximum pump power of $120.3 \mathrm{~W}$. The beam profiles of the $\mathrm{LP}_{11}$ mode are well maintained during the power amplification. The $3 \mathrm{~dB}$ linewidth of the $\mathrm{LP}_{01}$ and $\mathrm{LP}_{11}$ modes slightly increases from $\sim 0.23$ to $\sim 0.24 \mathrm{~nm}$ and from $\sim 0.24$ to $\sim 0.26 \mathrm{~nm}$, respectively.

The switching time between the $\mathrm{LP}_{01}$ and $\mathrm{LP}_{11}$ modes is an important aspect relating to the performance of the laser system, which is measured using a $2 \mathrm{GHz}$ oscilloscope combined with a $5 \mathrm{GHz}$ photodetector. The signal input window of the photodetector is covered with a pinhole and placed close to the lob center of the $\mathrm{LP}_{11}$ mode. Therefore, higher voltage reading in the oscilloscope should be expected when the laser outputs the $\mathrm{LP}_{11}$ mode. Here, we define the switching time as the time interval from the moment when the detected signal starts to change to the time when the signal starts to stabilize. When the AIFG is outside the seed laser oscillator, as for the setup in Fig. 1(a), the switching time is mainly determined by the transit time of the acoustic flexural wave propagating through the acousto-optic coupling region of the AIFG and establishing
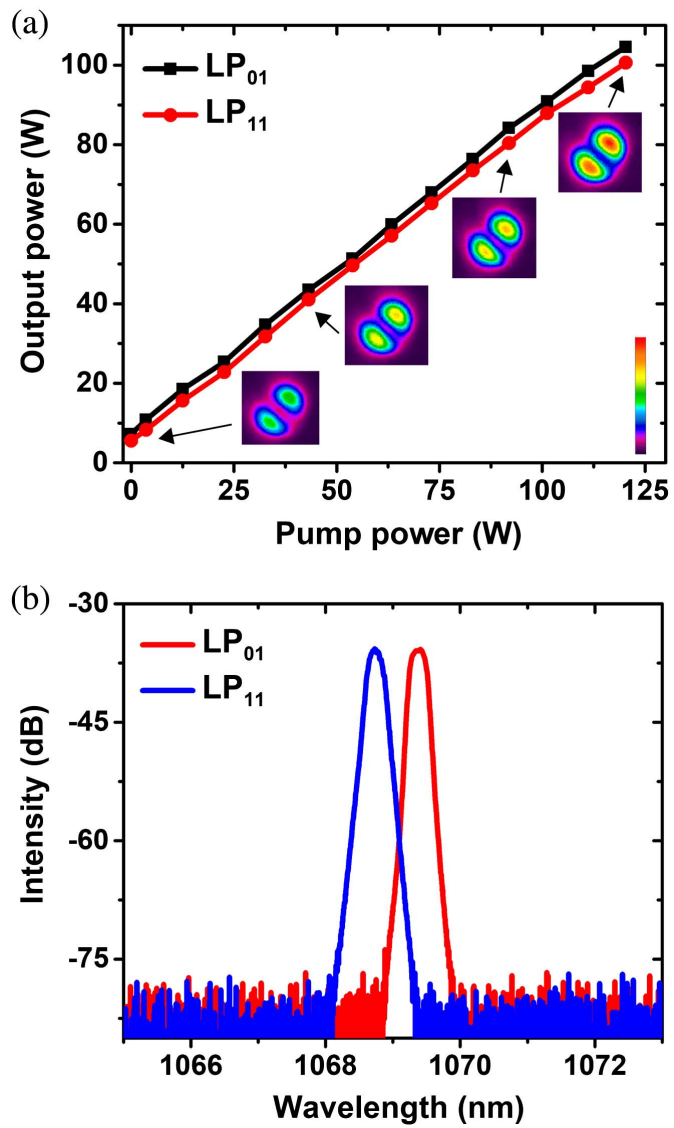

Fig. 5. (a) Output power of the $L P_{01}$ and $L P_{11}$ modes from the amplifier as a function of the pump power (insets: mode profiles of the $L P_{11}$ mode under different pump powers); (b) output spectra of the $L P_{01}$ and $L P_{11}$ modes under the maximum output power.

the standing wave, which is $\sim 0.5 \mathrm{~ms}$ in the current setup. The switching time from the $\mathrm{LP}_{01}$ to $\mathrm{LP}_{11}$ mode without the amplifier is measured to be $\sim 706 \mu \mathrm{s}$, and it takes $\sim 472 \mu$ s to switch back to the $\mathrm{LP}_{01}$ mode, the temporal traces of which are shown in Fig. 6(a). The actual switching time $(\sim 0.7 \mathrm{~ms})$ from the $\mathrm{LP}_{01}$ to $\mathrm{LP}_{11}$ mode is deviated from the theoretical value $(\sim 0.5 \mathrm{~ms})$, which could possibly be induced by the non-circularity of the GDF's inner cladding, and the residual strain in the unjacketed GDF during the AIFG fabrication. However, it would take a longer time after passing through the fiber amplifier since the saturation level of the amplifier also impacts the lasing dynamics, which reaches $\sim 963 \mu$ s from the $\mathrm{LP}_{01}$ to $\mathrm{LP}_{11}$ mode and $\sim 904 \mu \mathrm{s}$ from the $\mathrm{LP}_{11}$ to $\mathrm{LP}_{01}$ mode, as shown in Fig. 6(b). As for the setup shown in Fig. 1(b), the AIFG is integrated within the laser cavity, and mode switching is accompanied by wavelength shifting, which requires longer time for the oscillation buildup. As indicated by the temporal traces in Fig. 7(a), the switching time from the $\mathrm{LP}_{01}$ to $\mathrm{LP}_{11}$ mode is $\sim 878 \mu$ s and $\sim 982 \mu$ s the other way around in the oscillator. As presented in Fig. 7(b), the switching time in the amplifier also slightly increases to $\sim 950 \mu$ s from the $\mathrm{LP}_{01}$ to $\mathrm{LP}_{11}$ mode and $\sim 1012 \mu \mathrm{s}$ from the $\mathrm{LP}_{11}$ to $\mathrm{LP}_{01}$ mode, owing to the amplification 


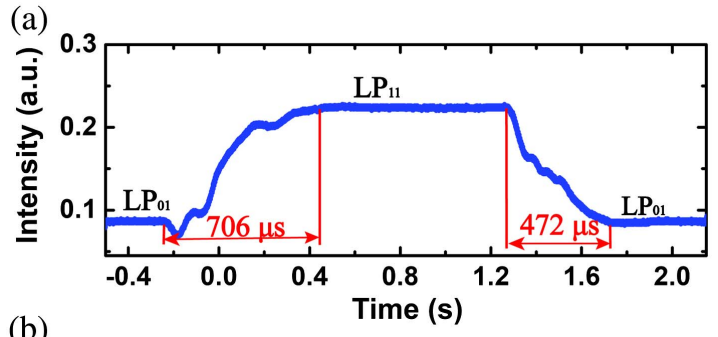

(b)

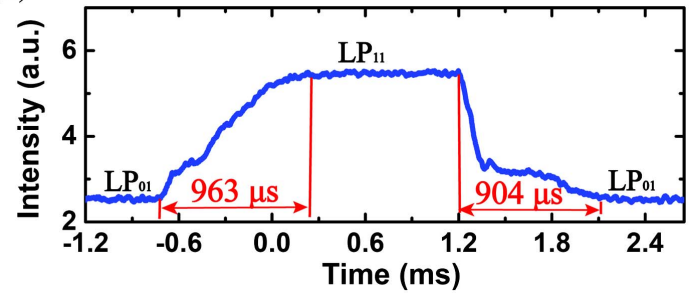

Fig. 6. (a) Switching time between the $L P_{01}$ and $L P_{11}$ modes of the seed laser with the AIFG outside the fiber oscillator; (b) switching time between the $L P_{01}$ and $L P_{11}$ modes from the amplifier with the AIFG outside the fiber oscillator.

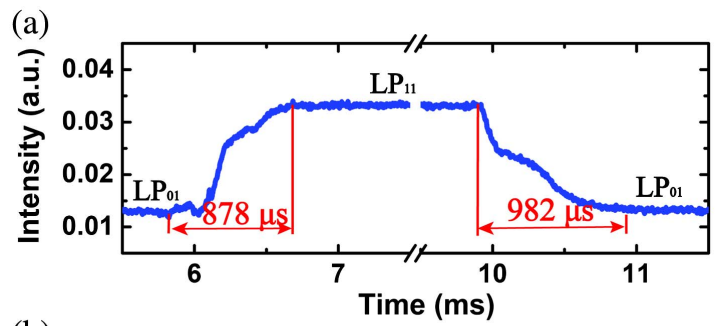

(b)

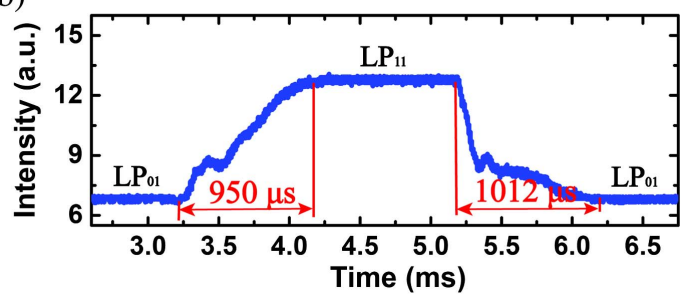

Fig. 7. (a) Switching time between the $L P_{01}$ and $L P_{11}$ modes of the seed laser with the AIFG inside the fiber oscillator; (b) switching time between the $L P_{01}$ and $L P_{11}$ modes from the amplifier with the AIFG inside the fiber oscillator.

saturation. Therefore, when the AIFG is outside the laser cavity, the switching time from the $\mathrm{LP}_{01}$ to $\mathrm{LP}_{11}$ mode is limited by the length of the acousto-optic interaction region, which could be reduced by employing shorter unjacketed GDF in the AIFG. However, according to our experimental results, when the mode switching is correlated with the wavelength switching process or when the laser extracts gain from the fiber amplifier, the mode switching speed is mainly limited by the lifetime of $\mathrm{Yb}^{3+}$ in the excited state, which is around $0.84 \mathrm{~ms}$. In this case, perhaps there is limited space for faster mode switching speed.

The stability of the output power of the $\mathrm{LP}_{11}$ mode in both configurations under the maximum pump power is also measured. The data are collected every $1 \mathrm{~min}$ for a total duration

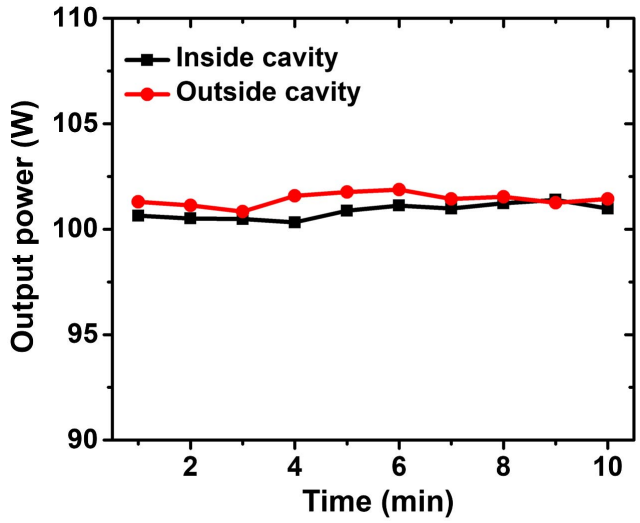

Fig. 8. Output power of the $L P_{11}$ mode in both configurations recorded in the total duration of $10 \mathrm{~min}$.

of $10 \mathrm{~min}$, as shown in Fig. 8. The output power of the MOPA configuration with AIFG inside the seed laser cavity ranges from 100.3 to $101.4 \mathrm{~W}$, and the maximum deviation of the output power from the average is $\sim 0.54 \%$. As for the configuration with AIFG outside the seed laser cavity, the output power from the amplifier fluctuates between 100.8 and $101.9 \mathrm{~W}$, with the maximum deviation of the output power from the average of $\sim 0.57 \%$. The power fluctuations in both configurations are very small, indicating good reliability of the system.

In comparison, although similar efficiencies, output powers, and switching times are obtained from the power amplifiers with two distinctively different mode-switchable seed lasers, quite different characteristics are found in these two seed lasers. The seed laser with the AIFG outside the cavity is superior in terms of switching time and overall efficiency owing to a simpler mode conversion process and lower insertion loss, which makes it more advantageous as the seed laser. In addition, it is also worth studying the mode purity difference with the AIFG inside and outside the laser cavity, which will be carried out through mode decomposition in the near future. In fact, since the AIFG is a passive component, it is also possible to realize a high-power mode switchable fiber laser with output power up to hundreds and even thousands of watts by integrating an AIFG directly after a high-power fiber laser.

Moreover, the fiber laser system adopting the AIFG can potentially be applied in the study of transverse mode instability (TMI). The TMI is recognized as the mode coupling process between the fundamental mode and high-order modes on the $\mathrm{kHz}$ level ${ }^{[35,36]}$. The AIFG, as a mode converter, can change the mode proportion on a $\mathrm{kHz}$ level, which could provide a controllable platform for the TMI study and suppression. Furthermore, by introducing closed-loop mode control of the AIFG in a high-power fiber laser system, an improved TMI threshold could possibly be achieved via $\mathrm{kHz}$ modulation of the pump sources or the signal lasers ${ }^{[37]}$. In addition, compared with the laser system based on the bulk-optics configuration in Ref. [37], the proposed fiber laser system integrated with AIFG features an all-fiber format, promising a more compact structure. 


\section{Conclusion}

In conclusion, we have demonstrated a high-power mode switchable fiber laser system based on an AIFG. Two kinds of several-watt-level mode switchable seed fiber lasers, with the AIFG inside and outside the laser cavity, are adopted as the seeds for power amplification. In comparison with all the previous intra-cavity mode switchable fiber lasers based on AIFG, a true few-mode fiber laser is realized with a designated setup, where the few-mode laser could extract the gain. Furthermore, by taking advantage of the MOPA technique, more than $100 \mathrm{~W}$ output power in both the $\mathrm{LP}_{01}$ and $\mathrm{LP}_{11}$ modes has been obtained. The MOPA system highlights the advantages of high-power capability up to hundreds and even thousands of watts, real-time mode switchability on the millisecond scale while operating in full power, and system simplicity without any free-space optical components for mode switching. Our work not only verifies the feasibility of realizing stable few-mode operation via dynamic mode switching in a fiber oscillator, but also realizes a high-power fiber amplifier with real-time mode switchability, which could find potential applications in material processing, TMI suppression, etc. Future work could be expected to realize mode switching between more transverse modes by adopting fibers with higher NA or larger core diameter and reach higher output power for more practical applications.

\section{Acknowledgement}

This work was supported in part by National Natural Science Foundation of China (No. 61805280), Open Research Fund of State Key Laboratory of Pulsed Power Laser Technology (No. SKL2020KF03), and Research Plan of National University of Defense Technology (No. ZK19-07). Part of this work has been accepted by the SPIE Photonics West 2022 Conference for oral presentation.

\section{References}

1. Y. Feng, L. R. Taylor, and D. B. Calia, " 25 W Raman-fiber-amplifier-based 589 nm laser for laser guide star," Opt. Express 17, 19021 (2009).

2. W. Shi, Q. Fang, X. Zhu, R. A. Norwood, and N. Peyghambarian, "Fiber lasers and their applications [Invited]," Appl. Opt. 53, 6554 (2014).

3. J. Zhao, G. Guiraud, C. Pierre, F. Floissat, A. Casanova, A. Hreibi, W. Chaibi, N. Traynor, J. Boullet, and G. Santarelli, "High-power all-fiber ultra-low noise laser," Appl. Phys. B 124, 114 (2018).

4. J. Shi, C. Li, H. Mao, Y. Ren, Z.-C. Luo, A. Rosenthal, and K. K. Y. Wong, "Grüneisen-relaxation photoacoustic microscopy at $1.7 \mu \mathrm{m}$ and its application in lipid imaging," Opt. Lett. 45, 3268 (2020).

5. R. Klas, A. Kirsche, M. Gebhardt, J. Buldt, H. Stark, S. Hädrich, J. Rothhardt, and J. Limpert, "Ultra-short-pulse high-average-power megahertz-repetition-rate coherent extreme-ultraviolet light source," PhotoniX 2, 4 (2021).

6. D. J. Richardson, J. Nilsson, and W. A. Clarkson, "High power fiber lasers: current status and future perspectives," J. Opt. Soc. Am. B 27, B63 (2010).

7. M. N. Zervas and C. A. Codemard, "High power fiber lasers: a review," IEEE J. Sel. Top. Quantum Electron. 20, 219 (2014).

8. P. Zhou, L. Huang, J. Xu, P. Ma, R. Su, J. Wu, and Z. Liu, "High power linearly polarized fiber laser: generation, manipulation and application," Sci. China Technol. Sci. 60, 1784 (2017).

9. https://www.coherent.com/lasers/fiber/highlight-fl-arm.
10. "Advanced metal processing-high-power fiber lasers with rogrammable beam technology," https://www.nlight.net/advanced-metal-processing.

11. https://www.spilasers.com/varimode/.

12. D. Mao, Y. Zheng, C. Zeng, H. Lu, C. Wang, H. Zhang, W. Zhang, T. Mei, and J. Zhao, "Generation of polarization and phase singular beams in fibers and fiber lasers," Adv. Photonics 3, 014002 (2021).

13. J. M. O. Daniel and W. A. Clarkson, "Rapid, electronically controllable transverse mode selection in a multimode fiber laser," Opt. Express 21, 29442 (2013).

14. X. Heng, J. Gan, Z. Zhang, J. Li, M. Li, H. Zhao, Q. Qian, S. Xu, and Z. Yang, "Transverse mode switchable all-fiber Brillouin laser," Opt. Lett. 43, 4172 (2018).

15. N. Wang, J. C. Alvarado-Zacarias, M. S. Habib, H. Wen, J. E. Antonio-Lopez, P. Sillard, A. Amezcua-Correa, A. Schülzgen, R. Amezcua-Correa, and G. Li, "Mode-selective few-mode Brillouin fiber lasers based on intramodal and intermodal SBS," Opt. Lett. 45, 2323 (2020).

16. Y. Cai, J. Wang, J. Zhang, H. Wan, Z. Zhang, and L. Zhang, "Generation of cylindrical vector beams in a mode-locked fiber laser using a mode-selective coupler," Chin. Opt. Lett. 16, 010602 (2018).

17. T. Wang, A. Yang, F. Shi, Y. Huang, J. Wen, and X. Zeng, "High-order mode lasing in all-FMF laser cavities," Photonics Res. 7, 42 (2018).

18. J. Lu, L. Meng, F. Shi, X. Liu, Z. Luo, P. Yan, L. Huang, F. Pang, T. Wang, $\mathrm{X}$. Zeng, and P. Zhou, "Dynamic mode-switchable optical vortex beams using acousto-optic mode converter," Opt. Lett. 43, 5841 (2018).

19. K. Wei, W. Zhang, L. Huang, D. Mao, F. Gao, T. Mei, and J. Zhao, "Generation of cylindrical vector beams and optical vortex by two acoustically induced fiber gratings with orthogonal vibration directions," Opt. Express 25, 2733 (2017).

20. W. Zhang, K. Wei, H. Wang, D. Mao, F. Gao, L. Huang, T. Mei, and J. Zhao, "Tunable-wavelength picosecond vortex generation in fiber and its application in frequency-doubled vortex," J. Opt. 20, 014004 (2017).

21. W. Zhang, L. Zhang, C. Meng, and F. Gao, "Generation of nanosecond cylindrical vector beams in two-mode fiber and its applications of stimulated Raman scattering," Chin. Opt. Lett. 19, 010603 (2021).

22. R. Su, B. Yang, X. Xi, P. Zhou, X. Wang, Y. Ma, X. Xu, and J. Chen, “ $500 \mathrm{~W}$ level MOPA laser with switchable output modes based on active control," Opt. Express 25, 23275 (2017).

23. Y. You, G. Bai, X. Zou, X. Li, M. Su, H. Wang, Z. Quan, M. Liu, J. Zhang, Q. Li, H. Shen, Y. Qi, B. He, and J. Zhou, "A 1.4-kW mode-controllable fiber laser system," J. Lightwave Technol. 39, 2536 (2021).

24. X. Du, H. Zhang, P. Ma, X. Wang, P. Zhou, and Z. Liu, "Spatial mode switchable fiber laser based on FM-FBG and random distributed feedback," Laser Phys. 25, 095102 (2015).

25. Y. Cai, Z. Wang, H. Wan, Z. Zhang, and L. Zhang, "Mode and wavelengthswitchable pulsed fiber laser with few-mode fiber grating," IEEE Photonics Technol. Lett. 31, 1155 (2019).

26. B. Sun, A. Wang, L. Xu, C. Gu, Z. Lin, H. Ming, and Q. Zhan, "Low-threshold single-wavelength all-fiber laser generating cylindrical vector beams using a few-mode fiber Bragg grating," Opt. Lett. 37, 464 (2012).

27. L. Huang, J. Leng, P. Zhou, S. Guo, H. Lu, and X. Cheng, "Adaptive mode control of a few-mode fiber by real-time mode decomposition," Opt. Express 23, 28082 (2015).

28. D. Lin, J. Carpenter, Y. Feng, S. Jain, Y. Jung, Y. Feng, M. N. Zervas, and D. J. Richardson, "Reconfigurable structured light generation in a multicore fibre amplifier," Nat. Commun. 11, 3986 (2020).

29. J. Song, H. Xu, H. Wu, J. Ye, J. Xu, L. Huang, J. Leng, and P. Zhou, "All-fiberized transverse mode-switching method based on temperature control," Appl. Opt. 58, 3696 (2019).

30. C. Jocher, C. Jauregui, M. Becker, M. Rothhardt, J. Limpert, and A. Tünnermann, "An all-fiber Raman laser for cylindrical vector beam generation,” Laser Phys. Lett. 10, 125108 (2013).

31. J. Lv, H. Li, Y. Zhang, R. Tao, Z. Dong, C. Gu, P. Yao, Y. Zhu, W. Chen, Q. Zhan, and L. Xu, "Few-mode random fiber laser with a switchable oscillating spatial mode," Opt. Express 28, 38973 (2020).

32. $\mathrm{H}$. Wu, J. Lu, L. Huang, X. Zeng, and P. Zhou, "All-fiber laser with agile mode-switching capability through intra-cavity conversion," IEEE Photonics J. 12, 1500709 (2020).

33. K. O. Hill and G. Meltz, "Fiber Bragg grating technology fundamentals and overview,” J. Lightwave Technol. 15, 1263 (1997). 
34. K. J. Lee, I.-K. Hwang, H. C. Park, and B. Y. Kim, "Axial strain dependence of all-fiber acousto-optic tunable filters," Opt. Express 17, 2348 (2009).

35. P. Ma, H. Xiao, D. Meng, W. Liu, R. Tao, J. Leng, Y. Ma, R. Su, P. Zhou, and Z. Liu, "High power all-fiberized and narrow-bandwidth MOPA system by tandem pumping strategy for thermally induced mode instability suppression,” High Power Laser Sci. Eng. 6, e57 (2018).
36. L. Xie, C. Zhang, Y. Liu, H. Li, Q. Chu, H. Song, W. Wu, B. Shen, M. Li, X. Feng, S. Huang, R. Tao, J. Wang, X. Zhang, and H. Zhu, "Experimental investigation of quasi-static mode degradation in a high power large mode area fiber amplifier,” Opt. Express 29, 7986 (2021).

37. H.-J. Otto, C. Jauregui, F. Stutzki, F. Jansen, J. Limpert, and A. Tünnermann, "Controlling mode instabilities by dynamic mode excitation with an acoustooptic deflector," Opt. Express 21, 17285 (2013). 